\title{
Boundary breaking for interdisciplinary learning
}

\author{
Adi Kidron* and Yael Kali \\ Technologies in Education Program, Faculty of Education, University of Haifa, Haifa, Israel
}

(Received 31 October 2014; final version received 27 September 2015)

The purpose of this work is to contribute to the body of knowledge on processes by which students develop interdisciplinary understanding of contents, as well as to suggest technology-enhanced means for supporting them in these processes in the context of higher education. In doing so, we suggest a rethinking of three traditional practices that tend to characterise typical higher education instruction: (1) compartmentalisation of disciplines; (2) traditional pedagogy; and (3) traditional hierarchies based on levels of expertise. Our high-level conjecture was that meaningful dialogue with peers and experts supports both the deepening of ideas in one knowledge domain and the formation of connections between ideas from several domains, both of which are required for the development of interdisciplinary understanding. We developed the Boundary Breaking for Interdisciplinary Learning (BBIL) model, which harnesses technology to break boundaries between disciplines, learners and organisational levels of hierarchy. Findings indicate that 36 undergraduate students who participated in an interdisciplinary online course that implemented the BBIL model have significantly improved their interdisciplinary understanding of the course contents. This study illustrates how innovative use of available, free and low-cost technology can produce a 'positive disruption' in higher education instruction.

Keywords: interdisciplinary learning; interdisciplinary understanding; learning community; cognitive apprenticeship; technology-enhanced learning; instructional design; higher education; online education

\section{Introduction}

The 21 st century and the 'knowledge revolution' pose challenges that demand different ways of thinking and the development of new skills. One of the critical skills is the ability to think and integrate knowledge across disciplines and to understand the relations between fields of knowledge (Frodeman 2010). Developing such an interdisciplinary understanding requires a learning process through which learners integrate insights and modes of thinking from a number of disciplines to advance their understanding of a topic which is beyond the scope of a single discipline. Boix-Mansilla (2010) refers to such a learning process as interdisciplinary learning. But when we turn to higher education institutions, as key players in preparing young people to cope with the challenges that this century poses, we find that although there are theories and pedagogical approaches that have the potential to promote interdisciplinary learning, it seems that current academic organisational structures are typically geared towards instruction that compartmentalises disciplines, instead of providing students with the

*Corresponding author. Email: adi.kidron@edtech.haifa.ac.il

Responsible Editor: Carlo Perrotta, University of Leeds, United Kingdom.

Research in Learning Technology 2015. (C) 2015 A. Kidron and Y. Kali. Research in Learning Technology is the journal of the Association for Learning Technology (ALT), a UK-based professional and scholarly society and membership organisation. ALT is registered charity number 1063519. http://www.alt.ac.uk/. This is an Open Access article distributed under the terms of the Creative Commons Attribution 4.0 International License (http://creativecommons.org/licenses/by/4.0/), allowing third parties to copy and redistribute the material in any medium or format and to remix, transform, and build upon the material for any purpose, even commercially, provided the original work is properly cited and states its license. 
tools for integrating knowledge (Salomon 1991). In fact, it is argued (e.g. by Christensen et al. 2011) that colleges and universities are in the midst of a complex crisis and are, therefore, expected to rethink their traditional goals and practices, in the face of competition from newer alternatives such as online education. Taking a disruptive innovation stance, one should 'rethink the age-old assumptions about higher education' (Christensen et al. 2011, p. 4). The current work suggests a rethinking of three traditional practices that tend to characterise typical higher-education instruction: (1) compartmentalisation of disciplines; (2) traditional pedagogy; and (3) traditional hierarchies based on levels of expertise.

The purpose of this work is to contribute to the body of knowledge that explains the processes by which students develop interdisciplinary understanding of contents, as well as to suggest technology-enhanced means for supporting students in these processes in the context of higher education. Our high-level conjecture (Sandoval 2014) is that interdisciplinary understanding entails a deep understanding of disciplinary ideas, simultaneously combined with the ability to see connections between different disciplinary ideas in several domains, and that these abilities are gained through meaningful dialogue and exposure to a diversity of ideas and ways of thinking.

In order to promote interdisciplinary understanding, we developed the Boundary Breaking for Interdisciplinary Learning (BBIL) model, which harnesses technology to address the limitations described above regarding compartmentalisation, traditional pedagogy and organisational hierarchies.

The BBIL model refers to three perspectives:

(1) From the curricular perspective, the model seeks to address the compartmentalisation challenge by technology-enhanced features, designed to promote interdisciplinary understanding and focusing on a cross-cutting theme to help learners integrate knowledge from several disciplinary lenses;

(2) From the pedagogical perspective, the model seeks to address the traditional pedagogy challenge by adopting a learning community approach (Bielaczyc, Kapur, and Collins 2013), in which a technological infrastructure is used for promoting a learning culture that enables participants to synthesise different views, solve problems and collaboratively advance knowledge using the wealth and diversity of ideas that community members contribute;

(3) From the organisational perspective, the model seeks to address the organisational hierarchy challenge by breaking the traditional boundaries between graduate and undergraduate students, while using technology-enhanced features that implement a cognitive apprenticeship approach (Collins 2006) to promote productive interactions.

This work focuses on the learning experience, as perceived by students who participated in academic courses in which the generic model was implemented, and on their learning outcomes with respect to interdisciplinary understanding.

\section{Background and related work}

\section{Breaking the boundaries between disciplines (the curricular perspective)}

In accordance with Thompson-Klein's (2010) taxonomy, we refer to 'breaking boundaries between disciplines' as interdisciplinarity, rather than multidisciplinarity. The main difference is that interdisciplinarity integrates various disciplinary perspectives 
to create new integrative knowledge, whereas multidisciplinarity combines disciplinary perspectives but with minimum interactions, while maintaining the identity of each discipline and its knowledge structures.

In order to explore the notion of interdisciplinarity, this work adopts the theoretical framework of Interdisciplinary Learning as a Pragmatic Constructionist View (ILPCV) (Boix-Mansilla 2010). Within this framework, interdisciplinary learning is a process by which learners integrate information, data, techniques, tools, perspectives, ideas, concepts and theories from two or more disciplines, to create products, explain phenomena, or solve problems in ways that would have been unlikely through single-disciplinary means. ILPCV describes four main cognitive processes by which learners integrate ideas from different disciplines and gradually develop interdisciplinary understanding:

(1) Establishing purpose (which involves creating a reference framework for the integration);

(2) Weighing disciplinary insights (including identification of relevant knowledge domains);

(3) Building leveraging integrations (by formulating connections and integrative insights);

(4) Maintaining a critical stance (by reconsidering and refining insights repetitively).

Another framework for exploring the integration of ideas is Knowledge Integration (KI) (Linn and Eylon 2011) which offers a conceptual and practical lens for understanding KI processes, for evaluating them and for supporting their emergence using instructional and design principles. KI focuses on the personal repertoire of ideas that students develop as a result of their learning experiences. By processing and creating links between these ideas, students build a coherent and normative understanding that enables them to interpret new situations. Linn and Eylon (2011) claim that these processes can be supported by eliciting students' ideas; adding new, pivotal ideas; developing criteria for distinguishing among ideas; and sorting out ideas.

\section{Breaking the boundaries between learners (the pedagogical perspective)}

As an educational approach, learning communities emphasises the social-cultural aspects of learning and the advancement of collective knowledge as a means for individual learning. A core characteristic of a learning community is the diversity of expertise among its members, who are valued for their contributions and are given support for personal growth and development (Bielaczyc, Kapur, and Collins 2013). The culture of a learning community encourages all participants to express their unique voices, as they bring their personal background and heritage into the discussion. Such a culture is gradually cultivated while breaking boundaries between individual learners. Technology has been shown to have an added value in supporting these processes (Kali, Levin-Peled, and Dori 2009; Scardamalia and Bereiter 1994). Hoadley (2012) describes three types of technology affordances for deploying or designing environments for communities. These include:

(1) A content affordance that refers to the representational capabilities that technology offers, including means for storing, manipulating and transmitting representations across distance or time; 
(2) A process affordance that refers to the capability of technology to enable scaffolding of tasks and activities;

(3) A context affordance that refers to the capability of technology to enable shifting between various social contexts (enabling virtual communication with a much broader audience, experiencing gender equity in an anonymous discussion, etc.).

\section{Breaking the boundaries between levels of organisational hierarchies (the organisational perspective)}

When considering learning within a community that brings together learners with different levels of expertise, the notion of cognitive apprenticeship (Collins 2006) becomes very relevant. The goal of cognitive apprenticeship, as a pedagogical approach, is to assist novices in gaining mastery in a certain skill or concept. The learning occurs in a natural context that involves both novices and experts, and is based on several core processes:

(1) Modelling: The expert performs a task so that novices can observe and build a conceptual model of the processes that are required for accomplishing the task;

(2) Coaching: The expert observes and facilitates while novices perform a task through which they exercise and practice;

(3) Scaffolding: The expert provides support to help the novice perform a task;

(4) Articulation: The expert encourages novices to verbalise their knowledge and thinking;

(5) Reflection: The expert enables novices to compare their own performance and progression with that of others;

(6) Exploration: The expert invites novices to present and solve their own problems.

The effectiveness of cognitive apprenticeship depends on the novices' ability to participate in the practices of their community. Lave and Wenger (1991) described this process as 'legitimate peripheral participation', in which novice members first practice peripheral tasks that do not require high levels of expertise, but enable them to enculturate and get familiar with the community's ways of thinking, doing and communicating. Nevertheless, these tasks, albeit peripheral, are necessary for the community's ongoing practice, and hence make the novices legitimate members. As the learning progresses, the novices gain expertise, practice core tasks and become more central members of the community.

The use of technology has been proven to augment cognitive apprenticeship processes and improve students' performance, as described in the work of Kopcha and Alger (2014) on Technology-Enhanced Cognitive Apprenticeship (TECA), which uses technology to enhance the processes, content and social aspects of cognitive apprenticeship. The researchers conclude that the TECA approach may be more beneficial when it coordinates multiple technologies that not only integrate key content with guidance and support for a variety of apprenticeship activities (e.g. modelling, coaching, scaffolding, reflecting), but also promote discussion of learning experiences in a larger community. And yet, as Hoadley (2012) describes, the notion of providing learners with access to experts, and the legitimacy for peripheral 
participation, stand in strong contradiction with the ways students are segmented into grades or levels within educational institutions. Furthermore, the traditional lecture format usually leaves no opportunity for students to participate in any practice related to the knowledge at hand. Thus implementing the notion of Breaking Boundaries between Organisational Hierarchies is likely to be a challenging task.

\section{Implications for design}

Connections between the above notions form the theoretical grounds for our generic model - a technology-enhanced learning community with varied levels of expertise can provide a rich environment for links to emerge between disciplines and for interdisciplinary understanding to develop among learners at all levels of expertise. Based on the three perspectives reviewed above, we defined the following 'pragmatic design principles' (Kali 2008), which we view as core principles for supporting interdisciplinary learning in higher education:

- Breaking Boundaries between Disciplines: The design should support learners in exploring ideas through both disciplinary and interdisciplinary perspectives. Technology-enhanced resources and activities should be developed to assist learners in deepening their understanding of the ideas within each of several disciplinary domains and for comparing and connecting ideas between the disciplines (Boix-Mansilla 2010).

- Breaking Boundaries between Learners: The design should promote a learning culture in which learners are motivated to develop their personal voice, form an opinion, feel comfortable to express ideas, respect other ways of thinking, negotiate ideas freely with learners at all levels of expertise and appreciate the potential of collaborative learning to enhance their own understanding (Bielaczyc, Kapur, and Collins 2013).

- Breaking Boundaries between Organisational Levels of Hierarchy: The design should support productive technology-enhanced interactions between learners at different levels of expertise; specifically, it should make visible and model expert thinking for novices, while coaching them as they gain higher levels of understanding (Collins 2006).

\section{Design}

The BBIL model is a generic model developed in this study and derived from the above pragmatic design principles. Technology plays a crucial role in embodying (Sandoval 2014) these design principles into the model's features; however, we only used available technologies, since we view the innovation of the BBIL model not in its cutting-edge technology, but rather in its conceptual design. Below, we describe the BBIL model and illustrate how we employed it for designing a set of two technologyenhanced semester-long higher education courses (undergraduate and graduate levels), in which students studied similar interdisciplinary contents adapted to their level of expertise. The courses also shared a similar title - Learning in a NetworKed Society (LINKS) - and involved six disciplinary knowledge domains each: learning sciences, science-communication, health sciences, cognition sciences, media and 
communication, and information sciences. The undergraduate course was taught fully online (learning environment developed in Moodle with embedded Google Docs), while the graduate course was taught in a hybrid format, with weekly meetings and online assignments (Google Sites with embedded Google Docs). Both courses were taught simultaneously (by the authors of this article) to enable interactions between them, as explained below.

Figure 1 illustrates the general structure of the two courses with a zoom-in into the technology-enhanced features used to create interactions between the two courses and the features designed for the learning of each disciplinary domain.

To implement the design principle of breaking boundaries between disciplines, the BBIL model includes the following design features:

(1) Cross-Cutting Theme: A theme that serves as a backbone through which knowledge from the different disciplines is integrated. The cross-cutting theme chosen for the LINKS courses was 'Learning in a Networked Society'. This theme was used for choosing the disciplinary resources (see Feature \#4) and for guiding the integrative artefacts (Feature \#2).

(2) Integrative artefacts: Following the study of more than one disciplinary domain, students are required to integrate ideas from these domains. Technology supports this integration by enabling the reuse of online artefacts created by students in earlier stages of the course (Kali et al. 2009), and the revisit of online discussions (Feature \#7). In the LINKS courses, the integrative artefact was an essay (1,000 words for undergraduates and 2,000 words for graduates), in which students were asked to answer one of several suggested interdisciplinary questions, integrating in their answers ideas from three disciplinary domains taught earlier in the course. Students submitted these essays and received feedback from instructors using Moodle's Assignment Module.

(3) Theme Lens: A set of generic questions that are derived from the cross-cutting theme and integrated into the online activities in each disciplinary domain

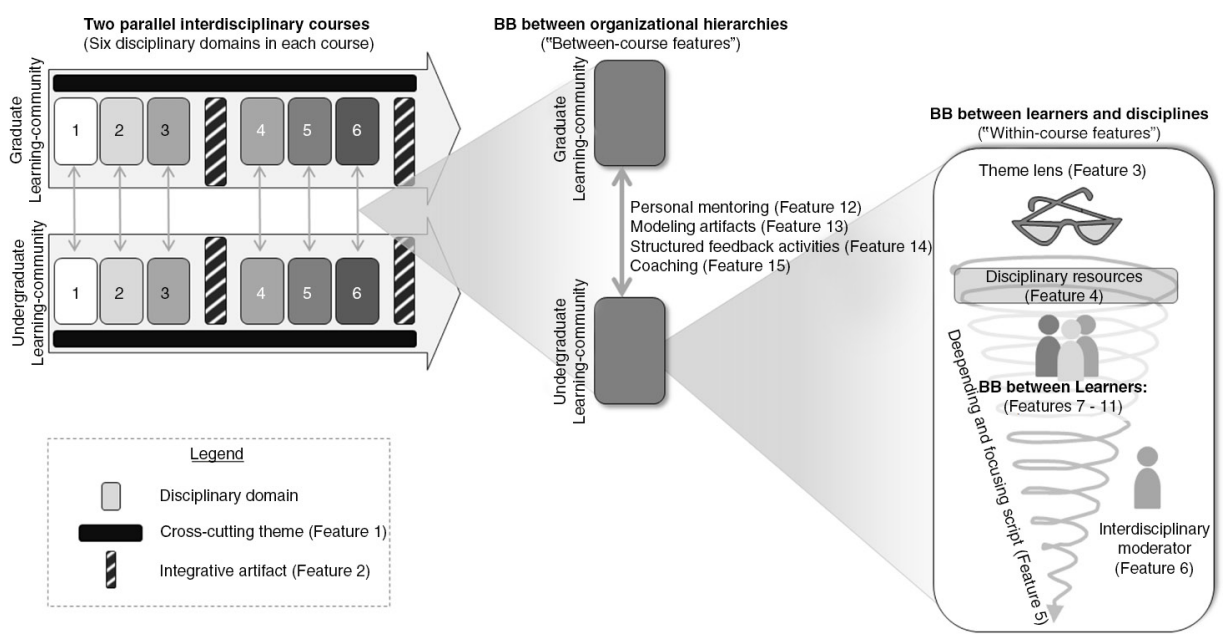

Figure 1. The BBIL model's structure and features (numbers of features refer to their description in the text). 
in order to promote the development of interdisciplinary connections. The Theme Lens for the two LINKS courses, which were used for the technologyenhanced collaborative knowledge-building activities (Feature \#7), were comprised of four various questions, such as 'How is learning conceptualised in this disciplinary domain?'

(4) Disciplinary Resources: Current technologies enable students' exposure to disciplinary experts and state-of-the-art knowledge artefacts (e.g. articles and video-recorded lectures that are uploaded to the course's website) in order to promote a thorough exploration of diverse disciplinary ideas. For each of the six disciplinary domains in the LINKS courses, we uploaded a videorecorded lecture and an article by an expert in that domain.

(5) Deepening and Focusing Script: A sequence of technology-enhanced activities, repeated for each disciplinary domain, were intended to deepen knowledge-building processes within the community and to lead the discussion gradually into the formation of connectable knowledge. We assumed that as students grew familiar with the script, they could focus their attention on the contents. This familiarity was supported by the repeated structure, interface and graphic design of the technological learning environment. In the LINKS courses, this included activities described in Features \#7, \#8, \#9 and \#10.

(6) Interdisciplinary Moderator: The moderator plays a critical role in the BBIL model - assisting the community of learners in delving deeply into each disciplinary domain and in making the connections between domains, to better understand the cross-cutting theme. The moderator's role, as we see it, does not necessarily require expertise in any of the disciplinary domains; however, interdisciplinary thinking, synthetic capabilities and moderating skills are prerequisites. The moderator can use various designated technological features as communication channels with the students (e.g. detailed feedback on the students' integrative assignments using Word's Comments and Track Changes features). In the LINKS courses, the instructor, who was a learning sciences researcher, undertook the interdisciplinary moderating role.

To implement the design principle of breaking boundaries between learners, the BBIL model includes the following design features:

(7) Collaborative Knowledge-Building Activities: Activities for learners to negotiate ideas and co-construct knowledge (using online collaborative tools, discussion boards, etc.). Several types of such collaborative knowledge-building activities (Bielaczyc, Kapur, and Collins 2013) were designed for the LINKS courses. For instance, one type of activity in the graduate level course utilised a pre-designed Google Docs file, which was simultaneously edited by all students in class, in order to answer the Theme Lens questions collaboratively (based on a previous activity in which disciplinary resources were discussed online in the community). Each team (about four students) was assigned one question, so that by the end of the session (about 30 minutes), the Google Docs text represented the ideas of the whole community regarding the four Theme Lens questions. This artefact was used further, as explained below. 
(8) Reuse of Student Artefacts: Sequenced activities in which artefacts that have been developed and saved in the course website in one stage are used by other members of the community at another stage in a learning sequence (Kali et al. 2009). In the LINKS courses, for instance, the Google Docs file designed to be co-constructed by teams in the graduate level course was later used by other teams to create a shared presentation that served as a modelling artefact for the undergraduate students (Feature \#13).

(9) Peer-Review Activities: Activities in which learners provide constructive, though critical, feedback to each other regarding course artefacts. In the LINKS courses, students had the opportunity to provide (and receive) peer feedback on their integrative essay (Feature \#2) before submitting it. Technology enabled the process to be self-monitored and managed by the students, who tracked versions and text changes of their work on the course website.

(10) Social Activities (related to disciplinary domains): Activities to support the development of personal acquaintance between learners within and between courses, which relate to the general contents of the disciplinary domains. Technology plays a crucial role in bridging time and distance gaps between students. In the LINKS courses, we designed 'Appetiser' activities that opened the learning sequence for each of the disciplinary domains, in which members of the two courses interacted unofficially with each other (asynchronously) regarding an issue related to the contents to be discussed later. For instance, the science-communication learning sequence, which focused on curricular design based on students' interests, began with an 'Appetiser' activity in which students used a template to fill in their 'dream curriculum' in a shared presentation and commented on each other's contributions (Figure 2).

(11) Learning Community Norm Prompts: Prompts designed to promote norms such as those described in the breaking boundaries between learners design principle, which are necessary for a productive learning community to develop (e.g. respect other ways of thinking and appreciate the potential of collaborative learning for enhanced understanding). In the LINKS undergraduate course, because it was fully online, weekly public messages were used as well as personal feedback on discussion posts (using Moodle's customisable evaluation rubrics), in which the instructor provided feedback not only on contents, but also on norms.

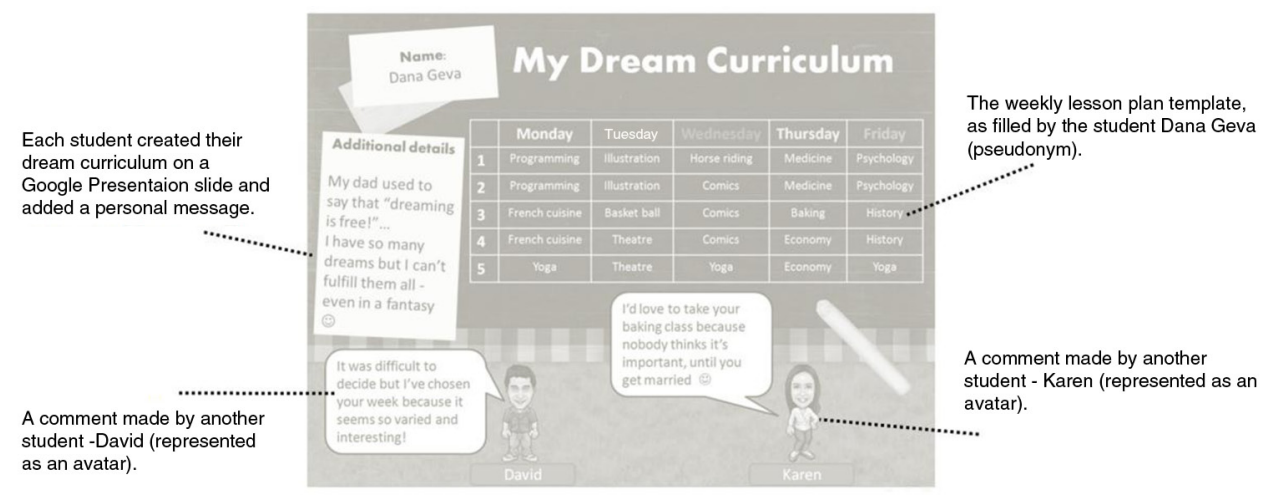

Figure 2. A screenshot taken from an 'Appetiser' activity (names have been changed for anonymity). 
To implement the design principle of breaking boundaries between levels of organisational hierarchy, the BBIL model includes the following features:

(12) Personal Mentoring: Technology-enhanced communication channels that enable personal mentoring of novices by advanced community members, with an emphasis on reflections about the learning process and enculturation, rather than on understanding the contents. In the LINKS courses, Moodle's Messages were used for this purpose.

(13) Modelling Artefacts: Technology-enhanced artefacts, developed by advanced community members to model their own ways of thinking, that are shared with novices. In the LINKS courses, these were the shared online presentations described above, which have been developed by graduated students in order to summarise their learning for each disciplinary domain. To streamline this process, Google Presentations that have been shared with all students and embedded in both courses' websites served as bridging artefacts between the courses.

(14) Structured Feedback Activities between Communities: Activities that enable feedback on artefacts that are shared between the communities. For the LINKS courses, we developed online forms for undergraduate students to provide feedback for graduate students regarding the modelling artefacts which they had shared with them. These forms were also embedded in both courses' websites and served as bridging artefacts.

(15) Coaching: Technology-enhanced infrastructures that enable advanced community members to assist novices in gradually developing more advanced knowledge and skills. In the LINKS courses, following permission from all undergraduate students, graduate students were provided with access to asynchronous discussions of the undergraduate course. For each disciplinary domain discussion, a team of graduate students was tasked with intervening by highlighting fruitful notions and directing the discussion.

\section{Methods}

This article reports our findings from an implementation of the two LINKS courses in the Spring semester of 2013, at one of the universities in Israel. We focused our analysis on the learning processes of students from the undergraduate course. The rationale for this decision was that unlike graduate courses, typical undergraduate courses at that university rarely use a learning community approach. Therefore, we assumed that the impact of our model on undergraduate students would be stronger and that their learning processes would be more salient and characterisable.

For methodological purposes, we define interdisciplinary understanding, operationally, as the ability to explain a cross-cutting theme in a coherent and logical way, using arguments that integrate knowledge from different disciplinary domains. Specifically, this study sought to answer two complementary questions:

(1) To what extent did students at the undergraduate level develop interdisciplinary understanding of the contents studied in the 'Learning in a Networked Society' course?

(2) How did they perceive the overall learning experience? 


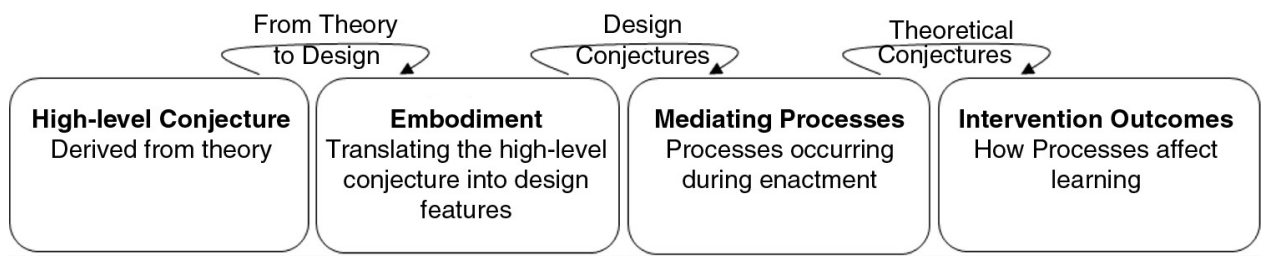

Figure 3. Generalised conjecture map (adapted from Sandoval 2014, in Kali, Sagy, Kuflik, Mogilevsky, and Maayan-Fanar 2015).

\section{Methodological approach}

Given the coupling of theoretical and design objectives in this research, a designbased research methodological approach was chosen. This approach was developed to enable systematic examination of learning processes that occur in real-world settings (such as classrooms), that are mediated through the use of environments (often enhanced by technology) which have been designed to promote learning, as well as our understanding of the learning processes (Kali 2008; Collins, Joseph, and Bielaczyc 2004). Design-based research often uses mixed-method approaches to characterise learning processes that occur in innovative environments, as well as to produce generalisations, using a combination of quantitative and qualitative means (Chi 1997).

We used conjecture mapping - a technique for representing conjectures in designbased research (Sandoval 2014), for representing the different conjectures that initiated this work (high-level conjectures); influenced the design (design conjectures); and supported the definition of the research questions and conclusions (theoretical conjectures). Figure 3 illustrates the constructs of a generalised conjecture map, as described by Sandoval.

Figure 4 illustrates the mapping of the conjectures of the current study using Sandoval's (2014) conjecture mapping technique.

\section{Participants}

Thirty-six undergraduate students from the Faculty of Education chose the course as part of their elective requirements. Thirty-four students completed the course

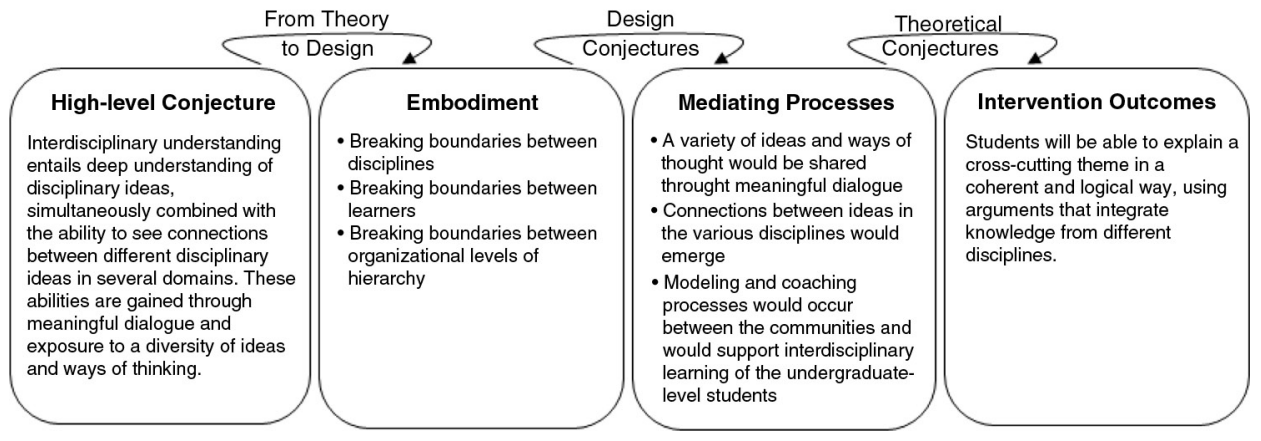

Figure 4. Conjecture mapping of the current research using Sandoval's (2014) technique. 
(a dropout rate of 5.5\%). Students varied in their academic background (e.g. theatre studies, history, music, psychology, literature, law, languages, communication, education, environmental studies) and had different professional experiences (teachers, social workers, lawyers, instructional designers). Previous experience with online courses varied from students with no experience at all, to students who had already participated in one or more online courses.

\section{Data sources}

\section{Interdisciplinary understanding (Research Question \#1)}

In order to evaluate the development of interdisciplinary understanding, data was collected through a task that was assigned twice, once in the middle of the course and once at the end. Students were required to choose one of several suggested questions regarding the cross-cutting theme and answer it individually in a 1,000-word essay, in which they were supposed to integrate ideas from the three disciplinary domains taught in the course (in the first assignment, this referred to the first three domains, whereas the final assignment referred to the other three). For instance, one of the questions was articulated as follows: 'In light of the three disciplinary domains recently presented in the course, what are the roles of teachers and students in a networked society, and how are they different from more traditional roles?'. All 34 students who completed the undergraduate course submitted the two essays $(100 \%$ of the participants).

\section{The perceived learning process (Research Question \#2)}

To shed light on the learning processes, as perceived by students, we presented a questionnaire at the end of the course with five open-ended questions that asked students about the course's contribution; the connection between the cross-cutting theme and the disciplinary contents; the added value of collaborative learning (as compared to individual learning); and their most and least meaningful design features. We also included one Likert-type question ('How would you describe your general learning experience in the course?') to assess the students' general satisfaction on a 1 ('very good') to 5 ('disappointing') scale. Of the 34 students in the course, 26 answered the questionnaire. The answers to the open question regarding the course contribution were detailed and, thus, provided rich data for analysis. Therefore, we segmented answers into statements that indicated various emergent themes. This resulted in 76 statements (an average of three statements per student), that were analysed, as described in the analysis section below.

\section{Data analysis}

\section{Interdisciplinary understanding}

Our assessment of interdisciplinary understanding was inspired by the KI framework (Linn and Eylon 2011). The original rubric is a 1-5 scale that assesses KI based on the number and quality of links between ideas, which students expressed in short answers they wrote in response to questions in specific knowledge domains. 
Certain adaptations were made to the original rubric, which referred to the following issues:

(1) The length of the student's response: The KI rubric was originally used for short answers, whereas we had long essays in the current study;

(2) The type of assignment: The KI rubric was originally used to analyse verbal explanations of a multiple-choice answer, whereas we needed it for a comprehensive, written, open answer;

(3) The content domain involved: The KI rubric was developed in the context of science learning, whereas we had to adapt it to the social sciences, in which canonical knowledge is more difficult to define;

(4) The types of links involved: The original KI rubric was developed to analyse links between ideas within the same knowledge domain, whereas the main objective of the current research was to analyse links between different knowledge domains.

Using the Adapted KI Rubric, two judges separately evaluated the mid-term and end-of-course assignments of all students. Each assignment was graded on a 1-3 scale in reference to three criteria: (1) Disciplinary grounding (profound and accurate understanding of the disciplinary ideas); (2) Integration (analysis of each disciplinary domain in light of the synthesis question, integration of the disciplinary ideas into a summarising answer); and (3) Quality of writing (coherency). After agreeing upon the score of each of the essays, only the integration scores were normalised to a $0-100$ scale, and an average mean integration score for each assignment was calculated. To compare the integration mean score of the mid-course assignment to the integration mean score of the final assignment, a paired-samples $t$-test was conducted.

\section{The perceived learning process}

Students' answers to the open-ended question were analysed using Chi's (1997) approach for quantifying qualitative analyses of verbal data. This approach includes four main stages. In the first stage (data volume reduction), we read all the answers and decided to focus on the analysis of one general question ('What did you gain from the course?'), in which students described their experience in their own words and expressed ideas that enabled us to infer about the mediating processes (Figure 4). Then, in the second stage (data segmentation), we decided that our unit of analysis would be a statement representing one main theme within a student's answer. Most of the answers included more than one theme. For instance, 'The course expanded my options of personal independent learning (categorised as belonging to the Added Value of Technology theme) and allowed me to experience thorough learning, collaborative learning and online interactions (the Community Culture theme), and enriched my knowledge allowing me to learn new topics (Knowledge and Skills theme)'. In the third stage (data coding), we developed the coding scheme presented in Table 1, based on emergent themes from the previous stage. Two judges separately used it for coding all of the students' answers. An agreement of $88 \%$ was reached when coded independently and 100\% agreement following discussion. Finally, in the fourth stage (pattern interpretation), we calculated the percentages of students whose statements have been coded for each theme. 


\section{Findings}

\section{Interdisciplinary understanding (Research Question \#1)}

Students' interdisciplinary understanding of the LINKS contents improved significantly; the $t$-test indicated a significant difference $[t(31)=2.96, p<0.01]$ in students' KI scores between the assays that they had written for the mid-course assignment $(M=67.2, \mathrm{SD}=29.4)$ and those they wrote for the final assignment $(M=82.5, \mathrm{SD}=22.0)$.

\section{The perceived learning experience (Research Question \# 2)}

Students rated highly their learning experience in the course, as indicated from the Likert-type question in the questionnaire $(M=4.4, \mathrm{SD}=0.69)$. Five main themes emerged from the analysis of students' answers to the open-ended question (Table 1). As represented in the table, though not directly asked about these themes, $73 \%$ of the students mentioned different aspects of the Knowledge and Skills theme, which they felt they had gained in the course; $50 \%$ of the students mentioned ideas that indicated their view of the course as disruptive due to its pedagogical and technological nature; $42 \%$ indicated the added value of technology; $27 \%$ pointed out that the culture of the learning community had been meaningful to their learning; and finally, $12 \%$ referred to the interactions with the graduate course students as an asset.

\section{Discussion and conclusion}

This work was initiated with our high-level conjecture (Sandoval 2014) that meaningful dialogue with peers and experts supports both a deepening into ideas in one knowledge domain and developing connections between ideas from several domains, which are both required for the development of interdisciplinary understanding. We assumed that the affordances of technology would support and enable these processes.

Our first research question focused on the intervention outcomes and sought to explore the extent to which interdisciplinary understanding can be developed and improved. The analysis and comparison between final and mid-term essays indicates that students who participated in the LINKS undergraduate course had significantly improved their interdisciplinary understanding of the cross-cutting theme. This theme - Learning in a Networked Society - which was also the topic of the course, was the main notion that we sought for students to develop their interdisciplinary understanding about. Thus, this research illustrates how careful design, based on theoretically grounded design principles and embodied through a technologyenhanced learning environment, can lead to interdisciplinary understanding, despite the challenges of supporting students in achieving such understanding, as described in the literature (e.g. Boix-Mansilla 2010). We argue that technology, and the unique ways in which it was employed in conjunction with the design principles of the BBIL model, had played a crucial role in this achievement. This claim is supported, as we illustrate below, by the way students perceived their learning experience.

Our second research question sought to explore the way in which students perceived the learning experience, in order to shed light on the mediating processes (Sandoval 2014) that had enabled this significant improvement. We argue, as explained below, that each of the boundary-breaking design principles, as they had been embodied through technological features in the LINKS courses, had contributed to 
Table 1. Main themes that emerged from analysis of the open question ('What did you gain from the course?').

\begin{tabular}{lll}
\hline Emergent theme from students' & $\begin{array}{l}\text { Percentage of } \\
\text { answers to the question 'What did } \\
\text { you gain from the course?' }\end{array}$ & $\begin{array}{l}\text { students who } \\
\text { referred to the } \\
\text { theme }\end{array}$ \\
\hline
\end{tabular}

(1) Knowledge and skills gained in the $73 \%(19 / 26)$ course

Course described as new, diverse, interesting and relevant topics; critical thinking, writing skills, time management, distance learning skills

(2) Disruptive nature of the course model

Course described as unique and unusual that changed students' perceptions about academic learning

$50 \%(13 / 26)$

12 positive statements; one negative statement

(3) Added value of technology Claims reflecting positive changes in attitudes towards technology and its potential in educational contexts; claims regarding contribution of technology to selfregulation and intrinsic motivation

(4) The community culture as a contribution to learning

Participating in a discussion, listening to other opinions, expressing opinions; social interactions; learning with others, exchanging ideas, cross-fertilisation

(5) The cognitive apprenticeship approach as an asset Learning from the graduate course's products, giving and receiving feedback

$12 \%(3 / 26)$
'The course topics were very interesting and appealing and encourage me to continue reading and exploring them'.

'The main contribution ... is the change in the way of learning. I realized there are other ways to teach an academic course. The course topics were taught in a way that made them interesting and meaningful for me'.

$42 \%(11 / 26) \quad$ 'At the beginning of the course I was very critical about online learning, but at the end I could see the advantages of such learning, and suddenly it was difficult to find any drawbacks'.

'I developed responsibility to track the course's lectures and assignments by myself'.

27\% (7/26) '[I learned] how to accept different ideas and other opinions which may be opposed to mine, how to articulate my own responses in a right way so that others would be able to accept them'.

${ }^{\text {a }}$ Note that this column adds up to more than $100 \%$ because students' answers typically included more than one theme.

these mediating processes. In fact, we view 'boundary-breaking' as a mindset that liberates thinking and promotes mutual growth and cross-fertilisation.

The strongest theme emerging from students' answers to the open-ended question regarding the contribution of the course was the gaining of deep diverse knowledge and important skills (Emergent Theme \#1). This finding becomes even stronger given the potential pitfall of shallowness or confusion when interdisciplinary instruction is implemented (Boix-Mansilla 2010). We believe that the features that were designed to break boundaries between disciplines - such as the Cross-Cutting Theme and the Theme Lens - created a framework into which students could integrate disciplinary 
ideas coherently and, therefore, develop deep understanding, as described in our mediating processes.

Regarding the breaking of boundaries between learners, an interesting finding is that the collaborative learning processes (categorised as Learning Culture in Emergent Theme \#4, e.g. exchanging ideas, exposure to a variety of ideas) seems to have had a meaningful contribution to the development of interdisciplinary understanding. We interpret these as representing one of the other mediating processes - meaningful dialogue and sharing of ideas - and argue that these processes are highly dependent on the technological affordances that were available to the students (e.g. overcoming time and distance gaps, co-constructing knowledge, sharing and recording personal ideas, revisiting discussions).

To implement our third boundary-breaking principle - between levels of hierarchy - we designed interactions between the undergraduate and graduate courses, which could only be enacted using technology-enhanced features. We conjectured that these would support another mediating process - modelling and coaching between the communities. The relatively low percentage of references that students from the undergraduate course made to these interactions (categorised as Cognitive Apprenticeship in Table 1) remains unexplained. A possible explanation is that this resulted from the phrasing of the question (which referred to the contribution of the course and not specifically to the processes and features that led to that contribution). Another possible explanation is that students might not have been aware of the impact of the cognitive apprenticeship's features on their learning. Either way, it seems that the set of technology-enhanced features that employed this design principle had not been sufficient to meaningfully support this expected mediating process and should be improved and further studied in future implementations.

A most encouraging finding was the students' perceptions of the disruptive nature of their learning experience in the course. Although students were asked about what they gained from the course (and not what they thought about it in comparison to other courses), $50 \%$ of them chose to include statements that expressed the uniqueness of the model in comparison to their usual learning experience. In addition, the findings indicate that technology had played a crucial role in creating this positive disruption: Many of the students $(42 \%)$ positively changed their attitudes and came to recognise the added value of technology in promoting learning in general, and aspects such as self-regulation and motivation, in particular, through the distant-learning experience provided by the course. Combined with students' high ratings of their general experience in the course (indicated by their answers to the Likert question), we interpret these findings as indicating that the model, as a whole, and the use of technology had succeeded in creating a very positive disruption in the way students perceived learning in higher education.

With regards to the role of technology, we argue that it played a critical role in implementing our model. Many of the features designed for the course could not have been realised, or would have been very difficult to implement, without the use of technology. This includes features from all of the three boundary-breaking principles: the video-recorded lectures that served as disciplinary resources (Feature \#4); the wholeclass simultaneous editing of collaborative documents that served for knowledgebuilding activities (\#7); the reuse of these documents for developing modelling artefacts (\#8); the tracking of versions and text changes in documents for the peerreview activities (\#9); the streamlining of modelling artefacts using collaborative documents shared by students in the two courses (\#13); the online forms for 
structured feedback activities between communities (\#14); and the coaching that was provided by enabling graduate students for intervening in the undergraduate course's online discussions (\#15). Thus, this study illustrates that the heart of innovation in educational technology is not necessarily dependent on cutting-edge technology. Innovative use of available, free and low-cost technology is the key to introducing disruptive learning experiences into higher education.

Finally, going back to the challenges posed by Christensen et al. (2011), we argue that this work exemplifies a solid educational basis for rethinking traditional goals, practices, essence and purpose of teaching in higher education. Though this work describes a particular implementation within specific disciplinary domains, the BBIL model has been designed as a generic model, so that its principles can be adopted and implemented in any set of disciplinary domains. We believe that the positive disruption that it created in the current study can be reproduced and thus, redefine the quality of education in other higher education terrains.

\section{Acknowledgements}

This research was supported by the I-CORE Program of the Planning and Budgeting Committee and The Israel Science Foundation grant 1716/12. The authors would like to thank Shai Spieler and his team from the University of Haifa eLearning Support Unit for their help and support.

\section{References}

Bielaczyc, K., Kapur, M. \& Collins, A. (2013) 'Cultivating a community of learners in K-12 classrooms', in International Handbook of Collaborative Learning, eds C. E. Hmelo-Silver, C. A. Chinn, C. K. K. Chan \& A. O'donnell, Routledge, New York, pp. 233-249.

Boix-Mansilla, V. (2010) 'Learning to synthesize: the development of interdisciplinary understanding, in The Oxford Handbook of Interdisciplinarity, eds R. Frodeman, J. Thompson-Klein, C. Mitcham \& J. B. Holbrook, Oxford University Press, Oxford, pp. 288-306.

Chi, M. T. H. (1997) 'Quantifying qualitative analyses of verbal data: a practical guide', Journal of the Learning Sciences, vol. 6, no. 3, pp. 271-315.

Christensen, M. C., et al., (2011) Disrupting College - How Disruptive Innovation Can Deliver Quality and Affordability to Postsecondary Education, Center for American Progress and Innosight Institute [online], Available at: http://files.eric.ed.gov/fulltext/ED535182.pdf

Collins, A. (2006) 'Cognitive apprenticeship', in The Cambridge Handbook of the Learning Sciences, ed R. K. Sawyer, Cambridge University Press, New York, pp. 47-60.

Collins, A., Joseph, D. \& Bielaczyc, K. (2004) 'Design research: theoretical and methodological issues', Journal of the Learning Sciences, vol. 13, no. 1, pp. 15-42.

Frodeman, R. (2010) 'Introduction', in The Oxford Handbook of Interdisciplinarity, eds R. Frodeman, J. Thompson-Klein, C. Mitcham \& J. B. Holbrook, Oxford University Press, Oxford, pp. 29-39.

Hoadley, C. (2012) 'What is a community of practice and how can we support it?', in Theoretical Foundations of Learning Environments, eds D. Jonassen \& S. Land, Routledge, New York, pp. 287-300.

Kali, Y. (2008). 'The design principles database as means for promoting design-based research', in Handbook of design research methods in education: Innovations in science, technology, engineering, and mathematics learning and teaching, eds A. E. Kelly, R. A. Lesh \& J. Y. Baek, Lawrence Erlbaum Associates, New Jersey, pp. 423-438.

Kali, Y., Levin-Peled, R. \& Dori, Y. (2009) 'The role of design-principles in designing courses that promote collaborative learning in higher-education', Computers in Human Behavior, vol. 5, pp. 1067-1078. 
Kali, Y., Sagy, O., Kuflik, T., Mogilevsky, O. \& Maayan-Fanar, E. (2015) 'Harnessing technology for promoting undergraduate art education: a novel model that streamlines learning between classroom, museum and home', IEEE Transactions on Learning Technologies, vol. 8, no. 1, pp. 5-17.

Kopcha, T. J. \& Alger, C. (2014) 'Student-teacher communication and performance during a clinical experience supported by a technology-enhanced cognitive apprenticeship', Computers \& Education, vol. 72 , pp. 48-58.

Lave, J. \& Wenger, E. (1991) Situated Learning: Legitimate Peripheral Participation, Cambridge University Press, Cambridge.

Linn, M. C. \& Eylon, B. S. (2011) Science Learning and Instruction: Taking Advantage of Technology to Promote Knowledge Integration, Routledge, New York.

Salomon, G. (1991) 'From theory to practice: the international science classroom - a technology-intensive, exploratory, team-based and interdisciplinary high school project', Educational Technology, vol. 31, no.3, pp. 41-44.

Sandoval, W. (2014) 'Conjecture mapping: an approach to systematic educational design research', Journal of the Learning Sciences, vol. 23, no.1, pp. 18-36.

Scardamalia, M. \& Bereiter, C. (1994) 'Computer support for knowledge building communities', The Journal of the Learning Sciences, vol. 3, no. 3, pp. 265-283.

Thompson-Klein, J. (2010) 'A taxonomy of interdisciplinarity', in The Oxford Handbook of Interdisciplinarity, eds R. Frodeman, J. Thompson-Klein, C. Mitcham \& J. B. Holbrook, Oxford University Press, Oxford, pp. 15-30. 